\title{
Article
}

\section{A-B Diblock Copolymer Micelles: Effects of Soluble-Block Length and Component Compatibility}

Yu-Jane Sheng, Tzu-Yu Wang, Wei Ming Chen, and Heng-Kwong Tsao

J. Phys. Chem. B, 2007, 111 (37), 10938-10945 • DOI: 10.1021/jp073408s

Downloaded from http://pubs.acs.org on November 24, 2008

\section{More About This Article}

Additional resources and features associated with this article are available within the HTML version:

- $\quad$ Supporting Information

- $\quad$ Access to high resolution figures

- $\quad$ Links to articles and content related to this article

- $\quad$ Copyright permission to reproduce figures and/or text from this article

\section{View the Full Text HTML}

\section{ACS Publications}




\title{
A-B Diblock Copolymer Micelles: Effects of Soluble-Block Length and Component Compatibility
}

\author{
Yu-Jane Sheng, $†$ Tzu-Yu Wang, ${ }^{\ddagger}$ Wei Ming Chen,, and Heng-Kwong Tsao ${ }^{*, \hbar, \S}$ \\ Department of Chemical Engineering, National Taiwan University, Taipei, Taiwan 106, Republic of China, and \\ Department of Chemical and Materials Engineering and Institute of Materials Science and Engineering, \\ National Central University, Jhongli, Taiwan 320, Republic of China
}

Received: May 4, 2007; In Final Form: July 12, 2007

\begin{abstract}
Micellization of a diblock copolymer in dilute solution is studied by dissipative particle dynamics. The influence of the compatibility between blocks $\mathrm{A}$ and $\mathrm{B}$ and the interaction between the insoluble block and solvent on aggregation number $P$ and micellar core radius $R_{\mathrm{c}}$ are examined. The micelle size distribution is obtained, and it is quite polydisperse. Different from the scaling theory for starlike micelles, the mean aggregation number based on weight average $\langle P\rangle_{\mathrm{w}}$ decreases with increasing soluble-block length $N_{\mathrm{A}}$ and the power law relation can be obtained, $\langle P\rangle_{\mathrm{w}} \sim N_{\mathrm{A}}^{-\alpha}$. Similarly, the micellar core radius declines with $N_{\mathrm{A}}$, following $R_{\mathrm{c}} \sim$ $N_{\mathrm{A}}^{-\beta}$ with $\beta=\alpha / 3$. However, the exponent depends on the mutual compatibility between soluble and insoluble blocks. For the same composition, the incompatible diblocks form a smaller micelle and its aggregation number declines with a smaller exponent $\alpha$. When $N_{\mathrm{A}} \sim N_{\mathrm{B}}$, the micelles deviate significantly from the spherical shape and solvophilic blocks are observed to be entrapped in the solvophobic core for compatible diblocks.
\end{abstract}

\section{Introduction}

Amphiphilic diblock copolymers in a selective solvent spontaneously self-assemble into supramolecular aggregates known as micelles, which are of fundamental interest and are important for dispersant technology, nanomaterial synthesis, and controlled release. ${ }^{1}$ The structure of micelles usually exhibits a spherical geometry in which the insoluble blocks form a closely packed inner core and the soluble blocks construct an outer corona or shell swollen by the solvent. Essential characteristics associated with the micelle are the equilibrium aggregation number $(P)$, the radius of micellar core $\left(R_{\mathrm{c}}\right)$, and the overall radius $\left(R_{\mathrm{m}}\right)$.

On the basis of minimization of the free energy for micellization of the $\mathrm{AB}$ diblock in a selective solvent, a number of theoretical models were developed to predict the micellar properties and their dependence on characteristics of a diblock copolymer with $N_{\mathrm{A}}$ repeated units for a soluble block and $N_{\mathrm{B}}$ for an insoluble block. In general, there are two different approaches. The simple scaling models assume the micelles to be completely spherical and monodisperse. ${ }^{2-5}$ The micellar freeenergy is approximated as a sum of three contributions (due to the core, the corona, and the interface between them): $F_{\text {micelle }}$ $=F_{\text {core }}+F_{\text {corona }}+F_{\text {interface }}$ Micellization is driven by the system's tendency to lower its surface energy at the core/solvent interface. Typically, $F_{\text {interface }}$ is taken as the product of interfacial area and interfacial tension. For crew-cut micelles $\left(N_{\mathrm{B}} \gg N_{\mathrm{A}}\right)$, $F_{\text {interface }}$ is mainly counterbalanced by insoluble-block B stretching in the core $F_{\text {core }}$. On the other hand, for starlike micelles

* To whom correspondence should be addressed. E-mail address: hktsao@cc.ncu.edu.tw.

National Taiwan University.

$\doteqdot$ Department of Chemical and Materials Engineering, National Central University.

$\S$ Institute of Materials Science and Engineering, National Central University.
$\left(N_{\mathrm{A}} \gg N_{\mathrm{B}}\right), F_{\text {interface }}$ is essentially balanced by confinement of soluble-block $\mathrm{A}$ in the corona $F_{\text {corona. In these two limits, all }}$ scaling theories predict that aggregation number and micellar core radius depend mainly on the size of the insolubleblock B.

In the scaling approach, only dominant contributions to the total free-energy are considered and therefore the scaling relationship can be obtained analytically. However, if all energy terms are taken into account, numerical calculations have to be invoked and thus they can be referred to as the numerical approach. Constant coronal polymer density profiles are assumed, and the numerical results for micellar properties can also be correlated by the power law relation. ${ }^{6-9}$ Although most numerical approaches ${ }^{6-8}$ suggest none or very weak dependence of micellar characteristics on the size of the soluble A block, Nagarajan and Ganesh ${ }^{9}$ found that the soluble block can have a strong influence on the micellization behavior, which is polymer specific. There are six contributions to $F_{\text {micelle }}: F_{\text {core }}$ containing two terms accounting for deformation and mixing of block B, $F_{\text {corona }}$ including two terms describing deformation and mixing of block $\mathrm{A}$, one entropic term due to localization of diblock polymers at core and corona, and the driving force $F_{\text {interface. }}$ For the PEO-PPO-water system, $P \sim N_{\mathrm{A}}^{-0.51}$ and $R_{\mathrm{c}}$ $\sim N_{\mathrm{A}}^{-0.17}$ were obtained.

There have been several attempts made to examine the scaling relations proposed in the theories. In general, micellar characteristics are characterized by light scattering ${ }^{10}$ and small-angle neutron scattering ${ }^{11}$ (SANS) techniques. If the structure of the micelles could be locked in by appropriate methods such as photo cross-linking, then the micellar properties may be identified by transmission electron microscopy. ${ }^{12}$ Although some experimental results agree with the scaling theories, particularly $N_{\text {B }}$ dependence, it is obvious to see that some other results do not follow any of the existing scaling relations. For example, 
the $N_{\mathrm{A}}$ dependence has been clearly observed. A series of poly(styrene-4-vinylpyridine) (PS/4VP) copolymers in toluene ${ }^{13}$ have been studied by light scattering and the aggregation number exhibits a scaling relation, $P \sim N_{\mathrm{A}}^{-0.79}$. A series of PEO-PEP copolymers in water have been investigated by SANS, and the scaling relation of $P$ agrees with the theory of Nagarajan and Ganesh for short PEO blocks. Nonetheless, for longer PEO blocks, $N_{\mathrm{A}}$-independent behavior is observed. ${ }^{14}$

Micellization of block copolymers has been studied since the 1960s. However, the inconsistency among mean-field theories and experiments indicates the lack of systematic investigation based on mesoscopic level observation. Molecular simulation is able to provide more microscopic level information than experiment. Results obtained by mesoscale simulations may provide valuable insights and clarify the discrepancies. In fact, a large number of computer simulations of micelle formation have been reported in the literature. However, it has been realized that to obtain a well-equilibrated micellar size distribution, one requires large systems with a lot of micelles in them simulated over a sufficient time range. As a result, the copolymers get exchanged between the micelles often enough. ${ }^{15,16}$ To satisfy the equilibrium condition, short block copolymers as short as $N_{\mathrm{A}}+N_{\mathrm{B}}=4$ are used in coarse-grained models. ${ }^{15,16}$ In addition, the solvent effect is neglected. The building of mesoscale micellar solutions with the use of classical molecular dynamics (MD) simulations at atomic resolution is a challenge at present owing to the length and the time scales at which these phenomena can occur. On the other hand, mesoscopic simulations, such as dissipative particle dynamics (DPD), can treat a wide range of length and time scales by many orders of magnitude compared to those of atomistic simulations. Consequently, DPD is a more suitable approach to investigate the micellar properties and morphologies. ${ }^{17,18}$

In this work, we perform DPD to systematically explore the micellar characteristics in dilute solutions. The distribution of the aggregation number of a micelle is obtained to examine the polydispersity. In addition to the scaling relation associated with soluble-block length, the influence of the compatibility between blocks $\mathrm{A}$ and $\mathrm{B}$ and the interaction between insoluble block and solvent on aggregation number and micellar core radius are studied. The asphericity of the overall micelle and micellar core is also calculated to illustrate the characteristics of micellar morphology, which are influenced by $\mathrm{A}-\mathrm{B}$ mutual compatibility and soluble-block length.

\section{Model and Simulation Methods}

The DPD method, introduced by Hoogerbrugge and Koelman in $1992,{ }^{19}$ is a particle-based, mesoscale simulation technique. This method combines some of the detailed description of the MD but allows the simulation of hydrodynamic behavior in much larger, complex systems up to the microsecond range. In a DPD simulation, a particle having mass $m_{i}$ represents a block or cluster of atoms or molecules moving together in a coherent fashion. These DPD particles are subject to soft potentials and governed by predefined collision rules. Like MD, the DPD particles obey Newton's equation of motion

$$
\frac{\mathrm{d} \mathbf{r}_{i}}{\mathrm{~d} t}=\mathbf{v}_{i}, \quad m_{i} \frac{\mathrm{d} \mathbf{v}_{i}}{\mathrm{~d} t}=\mathbf{f}_{i}
$$

where $\mathbf{f}_{i}$ denotes the total forces acting on particle $i$. The interparticle force $\mathbf{F}_{i j}$ exerted on particle $i$ by particle $j$ is made of a conservative term $\left(\mathbf{F}_{i j}^{\mathrm{C}}\right)$, a dissipative term $\left(\mathbf{F}_{i j}^{\mathrm{D}}\right)$, and a random term $\left(\mathbf{F}_{i j}^{\mathrm{R}}\right)$. In the DPD simulation, a special "DPD thermostat" for the canonical ensemble is implemented by using dissipative and random pairwise forces. As a result, the momentum is locally conserved, which leads to the emergence of hydrodynamic flow effects on the macroscopic scale. The total force acting on particle $i$ is then

$$
\mathbf{f}_{i}=\sum_{i \neq j}\left(\mathbf{F}_{i j}^{\mathrm{C}}+\mathbf{F}_{i j}^{\mathrm{D}}+\mathbf{F}_{i j}^{\mathrm{R}}\right)
$$

The sum acts over all particles within a cutoff radius $r_{\mathrm{c}}$ beyond which the forces are neglected. The size of a DPD particle can be characterized by $r_{\mathrm{c}}$. Since a DPD particle represents a cluster of molecules, $r_{\mathrm{c}} \approx O(1-10) \mathrm{nm}$. Typically, the conservative force is represented by a soft-repulsive interaction

$$
\mathbf{F}^{\mathrm{C}}\left(r_{i j}\right)=a_{i j} \max \left\{1-\frac{r_{i j}}{r_{\mathrm{c}}}, 0\right\} \mathbf{e}_{i j}
$$

where $r_{i j}$ is the distance between particles and $\mathbf{e}_{i j}$ the unit vector in the direction of the separation. The interaction parameter $a_{i j}$ represents the maximum repulsion between particle $i$ and $j$. The relationship between $a$ and the Flory-Huggins parameter $\chi$ has been established by Groot and Warren, ${ }^{20}$

$$
a_{i j}=a_{i i}+3.497 \chi_{i j}
$$

for density $\rho=3$, which corresponds to three DPD particles in a cube with length equal to the diameter of the particle. For all the simulations presented here, the units are conveniently taken as $m=k_{\mathrm{B}} T=r_{\mathrm{c}}=1$.

The $a_{i i}$ term is determined such that the compressibility of pure component $i$ is reproduced $\left(a_{i i} \approx 75 / \rho\right)$. Thus, $a_{i i}=25$ leads to the compressibility of water. If species $i$ and $j$ are fairly compatible, $\chi_{i j}=0$ and $a_{i j}=25$. As incompatibility between $i$ and $j$ increases, $a_{i j}$ increases. In the system, there are three different species of DPD particles, including solvent (S), hydrophilic particle (A), and hydrophobic particle (B). A diblock copolymer is divided into $N_{\mathrm{A}}$ solvophilic particles and $N_{\mathrm{B}}$ solvophobic particles. The repulsive interactions between the same species are set as $a_{\mathrm{SS}}=a_{\mathrm{AA}}=a_{\mathrm{BB}}=25$. In the study of the dependence of soluble-block length, the parameters are assumed $a_{\mathrm{AS}}=26$ and $a_{\mathrm{BS}}=40$, respectively, for interactions of solvent with solvophilic and solvophobic particles. We have chosen $a_{\mathrm{AB}}=27$ and $a_{\mathrm{AB}}=50$ to represent the conditions of mutual compatibility and incompatibility between $\mathrm{A}$ and $\mathrm{B}$ blocks. A diblock copolymer with polystyrene (PS) and poly4-hydroxystyrene (PHS) blocks is a typical example of copolymer showing good compatibility. PS is hydrophobic, and PHS is hydrophilic due to the hydroxyl group, but PS and PHS are fairly compatible for the similarity in their molecular structures. On the other hand, polystyrene- $b$-poly(acrylic acid) is a representative example of copolymer of incompatibility.

A polymer is modeled by connecting ordinary DPD particles with springs. Thus, additional spring forces $F^{\mathrm{S}}$ act between adjacent particles in the chain. The present work adopts a harmonic spring

$$
\mathbf{F}_{i j}^{\mathrm{S}}=C\left(r_{i j}-r_{0}\right) \mathbf{e}_{i j}
$$

with $C=100$ and $r_{0}=0.7$. Note that eq 5 is used to impose connectness within beads of a polymer, and the choice of $C$ and $r_{0}$ will not affect the qualitative behavior of the system studied in our work.

The forces $\mathbf{F}^{\mathrm{D}}$ and $\mathbf{F}^{\mathrm{R}}$ are responsible for the conservation of the total momentum in the system. The dissipative force denotes 
friction between the particles and explains energy loss. On the other hand, the random force incorporates the effects of Brownian motion into the larger length scale and heat up the system. They are coupled through the fluctuation-dissipation theorem and hence build a thermostat. ${ }^{21}$ The dissipative and the random force, respectively, have the forms

$$
\mathbf{F}_{i j}^{\mathrm{D}}=-\gamma \mathrm{w}^{\mathrm{D}}\left(\mathbf{r}_{i j}\right)\left(\mathbf{e}_{i j} \cdot \mathbf{v}_{i j}\right) \mathbf{e}_{i j}
$$

and

$$
\mathbf{F}_{i j}^{\mathrm{R}}=\sigma \mathrm{w}^{\mathrm{R}}\left(\mathbf{r}_{i j}\right) \theta_{i j} \mathbf{e}_{i j}
$$

where $\mathbf{v}_{i j}=\mathbf{v}_{i}-\mathbf{v}_{j}$ and $\mathrm{w}^{\mathrm{D}}$ (also $\mathrm{w}^{\mathrm{R}}$ ) is a $r$-dependent weight function chosen as

$$
\mathrm{w}^{\mathrm{D}}(r)=\left[\mathrm{w}^{\mathrm{R}}(r)\right]^{2}=\left\{(1-r)^{2}, r<1 \quad 0, r \geq 1\right.
$$

$\theta_{i j}$ is a randomly fluctuating variable with Gaussian statistics. A relationship is found between the amplitudes $(\gamma$ and $\sigma)$ and thermal energy $k_{\mathrm{B}} T$

$$
\sigma^{2}=2 \gamma k_{\mathrm{B}} T
$$

The dynamics of 81000 DPD particles, starting from random distribution, was simulated in a cubic box $\left(30^{3}\right)$ under periodic boundary conditions. The equation of motion is integrated with a modified velocity Verlet algorithm with $\Delta t=0.05$. A typical simulation required 300000 steps (about $1 \mu \mathrm{s}$ ) and the first 80000 steps are for equilibration. Note that the DPD simulation utilizes soft-repulsive potentials; therefore, the systems studied are allowed to evolve much faster than the "brute-force" molecular dynamics. A typical DPD simulation needs about (510) $\times 10^{4}$ steps to equilibrate. After that the resulting morphologies are analyzed. The micellar properties, equilibrium aggregation number $(P)$, radius of micellar core $\left(R_{\mathrm{c}}\right)$, and radius of overall micelle $\left(R_{\mathrm{m}}\right)$ are then determined every 2000 steps. For different compositions of the diblock copolymer, the DPD particle ratio of polymer to solvent is generally maintained at about 1:20 (4.8 vol\%).

Following the definition of mean molecular weight of polymers, the mean aggregate number $\langle P\rangle$ can be expressed as number average or weight average. That is, the mean property of a quantity $X$ can be expressed as number average $\langle X\rangle_{\mathrm{n}}$ or $\langle X\rangle_{\mathrm{w}}$, which is defined by

$$
\langle X\rangle_{\mathrm{n}}=\sum_{i} X_{i} n_{i}
$$

and

$$
\langle X\rangle_{\mathrm{w}}=\sum_{i} X_{i} w_{i}
$$

Here $n_{i}$ denotes the number fraction of the aggregate with aggregation number equal to $P_{i}$, and $w_{i}$ is the weight fraction of the $i$ th aggregate. Note that $\Sigma_{i} n_{i}=1, \Sigma_{i} w_{i}=1$, and all DPD particles have the same mass $m$. On the basis of experimental approaches, osmometry gives the former $\langle P\rangle_{\mathrm{n}}$ while light scattering yields the latter $\langle P\rangle_{\mathrm{w}}$.

Another quantity used to characterize the instantaneous shape of a micelle is asphericity $\delta$, which measures the deformation of the micellar morphology away from a spherical geometry. The asphericity $\delta$ is defined as 22,23

$$
\delta=1-3 \frac{\left\langle E_{2}\right\rangle}{\left\langle E_{1}\right\rangle^{2}}
$$

where $E_{i}$ are the respective invariants of the gyration tensor and are given by

$$
E_{1}=\lambda_{x}+\lambda_{y}+\lambda_{z} \text { and } E_{2}=\lambda_{x} \lambda_{y}+\lambda_{y} \lambda_{z}+\lambda_{x} \lambda_{z}
$$

where $\lambda_{x}, \lambda_{y}$, and $\lambda_{z}$ denote the three eigenvalues of the radius of gyration tensor of the aggregate. The value of $\delta$ varies from 0 to $1 ; \delta$ is equal to 0 for a perfect spherical globule, 0.25 for a circle, and 1 for a rod. ${ }^{24}$

\section{Results and Discussion}

A. Micelle Size Distribution. As mentioned earlier, the DPD simulation method allows systems studied to evolve much faster to equilibrium state than the traditional MD. Generally speaking, the system can be well equilibrated in less than 100000 steps. To examine whether the simulations in this work are completely equilibrated, Figure 1a shows the typical variation of the aggregation number $\langle P\rangle_{\mathrm{w}}$ with time for $\mathrm{A}_{10} \mathrm{~B}_{5}$. It can be seen that after 40000 time-steps, $\langle P\rangle_{\mathrm{w}}$ stays around a final value which clearly indicates that the system has reached equilibrium. Also, even after equilibrium, we have continually observed frequent exchanges of chains between micelles, and the outcome of this direct observation guarantees true equilibrium. We have also performed simulations with different initial conditions including starting from the final micellar configurations of different interaction parameters. The results are consistent for one another. The above mentioned situations are valid for all the systems studied in our work, since we have very short hydrophobic block $\left(N_{\mathrm{B}}=5\right)$ and long hydrophilic group $\left(N_{\mathrm{A}} \geq\right.$ $5)$. Thus, all the chains studied are quite mobile, and true equilibrium has been reached. However, we have done some tests for longer hydrophobic blocks $\left(N_{\mathrm{B}}>5\right)$ and it is noticeable that as $N_{\mathrm{B}}$ increases the micellar structures tend to be fixed without detectable exchanges of chains between aggregates.

Figure $1 \mathrm{~b}$ shows a snapshot of the dilute micellar solution for $\mathrm{A}_{10} \mathrm{~B}_{5}$, which consists of three isolated copolymers, small micelles $(P=2$ and 5), and large micelles $(P=16,21$, and $22)$. For the purpose of demonstration, the solvent and some micelles are removed. Evidently, micellar size distribution is not monodisperse at all. The polydispersity of micelles is manifested by the distribution of the aggregate number, $f(P)$, as demonstrated in Figure 1c. In addition to a lot of isolated copolymers, a wide distribution ranges from about $P=5$ to 30. For multidisperse micelles, it is difficult to choose a critical aggregation number $P_{\mathrm{c}}$ so that the micelle is defined as the copolymer aggregates with $P>P_{\mathrm{c}}$. Following the definition of mean molecular weight of polymers, the mean aggregate number $\langle P\rangle$ can be expressed as number average or weight average, as defined in eqs 10 and 11 . That is, the isolated copolymers are also counted for the mean aggregate number. For $\mathrm{A}_{10} \mathrm{~B}_{5}$ with incompatible diblocks $\left(a_{\mathrm{AB}}=50\right)$, we have $\langle P\rangle_{\mathrm{n}}=5.7$ and $\langle P\rangle_{\mathrm{w}}$ $=16.7$. Evidently, the contribution of isolated copolymers to weight-average aggregation number is relatively unimportant. The polydispersity index is a measure of the breadth of the distribution of aggregation number. The result $\langle P\rangle_{\mathrm{w}} /\langle P\rangle_{\mathrm{n}}=2.9$ also illustrates that a dilute diblock copolymer solution actually contains widely dispersed micelles.

Experimental studies of $\mathrm{A}-\mathrm{B}$ diblock copolymer micelles often gave average micellar size with standard deviation. The aggregation number is generally of the order of hundreds of chains and the size distribution is quite uniform. ${ }^{14}$ However, the typical aggregation number in our simulations is of the order of tens, which is much smaller than that observed in experiments. This relatively low mean aggregation number may be 

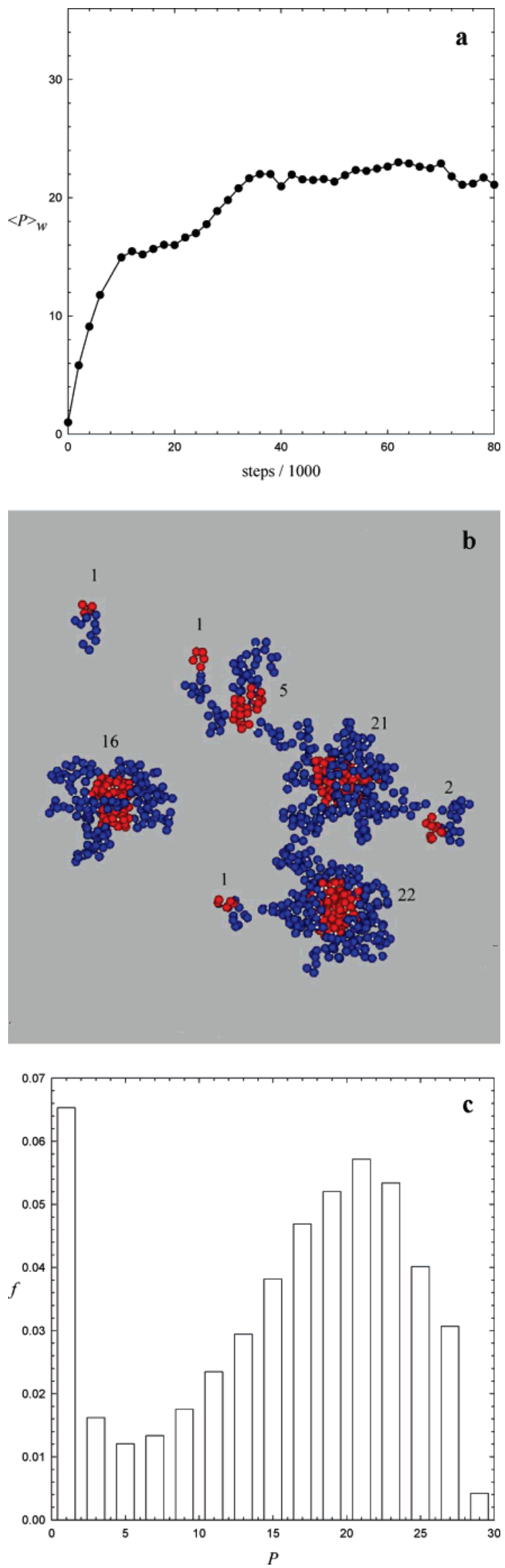

Figure 1. (a) The typical variation of the aggregation number with time for $A_{10} B_{5}$. (b) A snapshot of the dilute copolymer solution for $A_{10} B_{5}$. The number depicts the aggregation number of the micelle. (c) The distribution of the aggregate number for $\mathrm{A}_{10} \mathrm{~B}_{5}$.

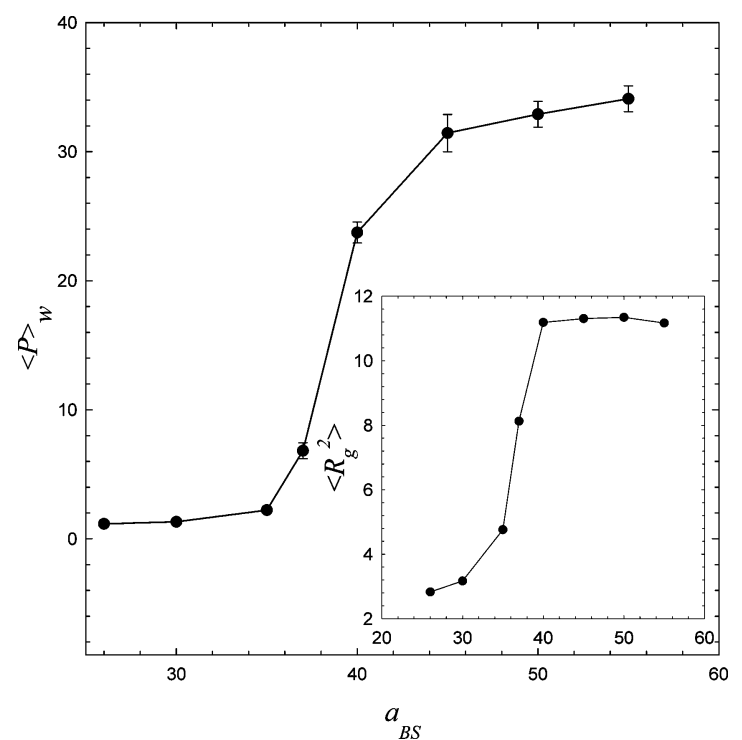

Figure 2. The variation of the weight-average aggregation number with the interaction parameter between insoluble $\mathrm{B}$ block and solvent $\left(a_{\mathrm{BS}}\right)$ for $\mathrm{A}_{10} \mathrm{~B}_{5}$ with $a_{\mathrm{AS}}=26$ and $a_{\mathrm{AB}}=40$. In the inset, the micellar core radius is plotted against $a_{\mathrm{BS}}$.

attributed to the low molecular weight of the coarse-grained model and the short insoluble length $N_{\mathrm{B}}=5$. The copolymers studied here are more like typical nonionic surfactants such as alkyl poly(ethylene oxide). For high hydrophobicity (long insoluble-block), however, the question of whether the observed aggregates were equilibrium structures still remains open. In a recent SANS study, the micelle structures initially formed upon dissolution are completely locked in for poly(ethylene oxide)polybutadiene block copolymers. ${ }^{25}$ No detectable exchange of chains between aggregates and solution was observed.

At the right balance of solvophilic and solvophobic moieties, diblock and triblock surfactants can spontaneously self-assemble into micelles. However, if one increases the solvophobic content of the molecule, macroscopic phase separation will occur. The schemes that allow us to distinguish between phase separation and micellization for diblock copolymer systems by simulations have been provided. ${ }^{26,27}$ As we have mentioned earlier, the copolymers studied in our work are more like typical nonionic surfactants, which are quite similar to the systems studied in these references. According to their work, the minimum ratio of hydrophilic to hydrophobic groups, $\mathrm{H} / \mathrm{T}$, required for formation of micelles decreases rapidly with increasing chain length. For $\mathrm{H}_{x} \mathrm{~T}_{y}$ diblock surfactants, this ratio ranges from 1 (for the shortest surfactants) to $1 / 4$ (for the longest). The ratios of hydrophilic to hydrophobic groups are all equal to or greater than 1 in our systems; therefore, it is unlikely for the phase separation to occur.

B. Solvent Quality and Mutual Compatibility. The ability for diblock copolymers to self-assemble into supramolecular aggregates depends on the compatibility between the solvophobic B-block and the solvent. Since all DPD particles are interacted by repulsive forces, a large (positive) value of $a_{i j}$ corresponds to strong repulsion between $i$ and $j$. As can be seen from Figure 2, by reducing the value of the interaction parameter $a_{\mathrm{BS}}$ (thereby increasing the compatibility between B block and solvents), the mean aggregation number of the micelle decreases and eventually only solitary diblock copolymers exist (i.e., $P$ $=1$ ). The radius of gyration of the micelle formed varies accordingly as shown in the inset. In Figure 2, there is a rather sharp transition for $a_{\mathrm{BS}}=35-40$, which indicates that largesize aggregates can only form when $a_{\mathrm{BS}} \gtrsim 40$ for $\mathrm{A}_{10} \mathrm{~B}_{5}$ with 


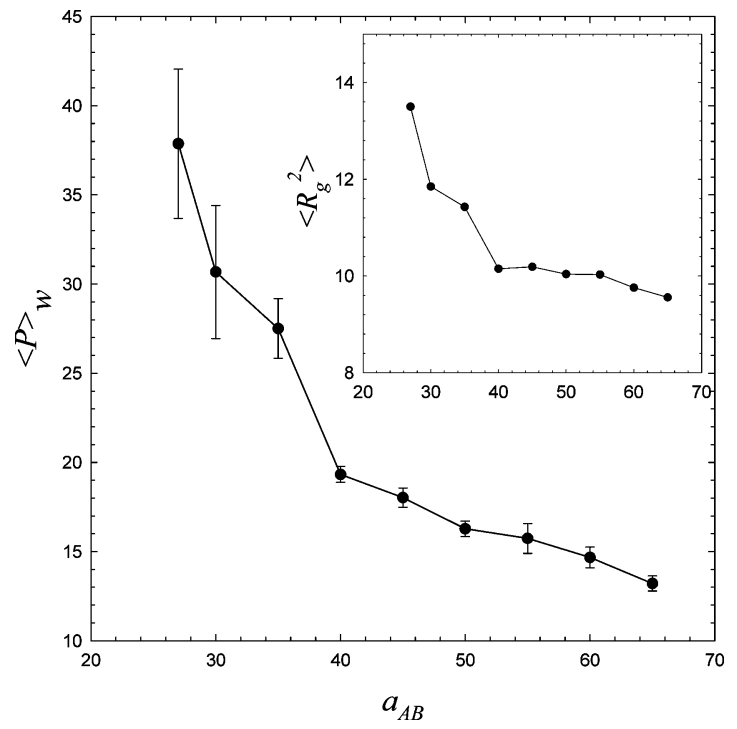

Figure 3. The variation of the weight-average aggregation number with the interaction parameter between soluble $\mathrm{A}$ block and insoluble B block $\left(a_{\mathrm{AB}}\right)$ for $\mathrm{A}_{10} \mathrm{~B}_{5}$ with $a_{\mathrm{AS}}=26$ and $a_{\mathrm{BS}}=40$. In the inset, the micellar core radius is plotted against $a_{\mathrm{AB}}$.

$a_{\mathrm{AS}}=26$ and $a_{\mathrm{AB}}=40$. This crossover value of $a_{\mathrm{BS}}$ is expected to vary with the length of solvophobic B blocks, and it is the outcome of the competition between the entropic (small $a_{\mathrm{BS}}$ ) and energetic (large $a_{\mathrm{BS}}$ ) effects. As B-block increases in length, it is anticipated that the crossover value of $a_{\mathrm{BS}}$ declines toward 25.

Most of the theoretical studies focus on investigating the effects of the physical properties of the solvophobic B block on the formation of the micelle. However, it is interesting to find that the mutual compatibility between the soluble and insoluble blocks also plays an important role in the micellar properties. Figure 3 demonstrates the variation of $\langle P\rangle_{\mathrm{w}}$ with $a_{\mathrm{AB}}$ for $\mathrm{A}_{10} \mathrm{~B}_{5}$, with $a_{\mathrm{AS}}=26$ and $a_{\mathrm{BS}}=40$. As one can see, $\langle P\rangle_{\mathrm{w}}$ increases as $a_{\mathrm{AB}}$ decreases. In other words, the aggregate grows bigger as $\mathrm{A}$ and $\mathrm{B}$ blocks become more and more compatible. For a given length of $\mathrm{B}$ block with fairly incompatible $\mathrm{A}-\mathrm{B}$ interaction, the micelle tends to be a typical spherical B-core and A-corona morphology as depicted in Figure 4 for $a_{\mathrm{AB}}=$ 50. There is a limitation as to the number of the insoluble $B$ blocks that a spherical core can accommodate. Therefore, $\langle P\rangle_{\mathrm{w}}$ gradually decays with increasing $a_{\mathrm{AB}}(z 40)$. However, as $a_{\mathrm{AB}}$ is small enough $(\$ 35)$, the core may deviate from the spherical shape. Figure 4 shows the variation of the asphericity $\delta$ with the mutual compatibility. For compatible $\mathrm{A}-\mathrm{B}$ blocks $\left(a_{\mathrm{AB}}=\right.$ 27 ), the asphericity increases from 0.09 to 0.2 , revealing a prolate-like micelle. The nonspherical shape of the aggregate can accommodate considerably more diblock copolymers than its spherical core-corona counterpart. Thus, $\langle P\rangle_{\mathrm{w}}$ increases noticeably.

Micellization is essentially driven by the reduction of the internal energy associated with the interactions between insoluble block and solvent. In the scaling approach, this contribution is represented by the surface free-energy (interfacial tension) associated with the interface between the core and the corona. In general, one expects that incompatible A-B monomers make contact at the core-corona interface and hence pay a surface energy cost. This surface energy should increase with both core-solvent repulsion $\left(a_{\mathrm{BS}}\right)$ as well as core-corona repulsion $\left(a_{\mathrm{AB}}\right)$. Since the aggregation number is proportional to the interfacial tension, the micellar size $P$ is anticipated to rise with $a_{\mathrm{AB}}$. However, our simulation results show that the

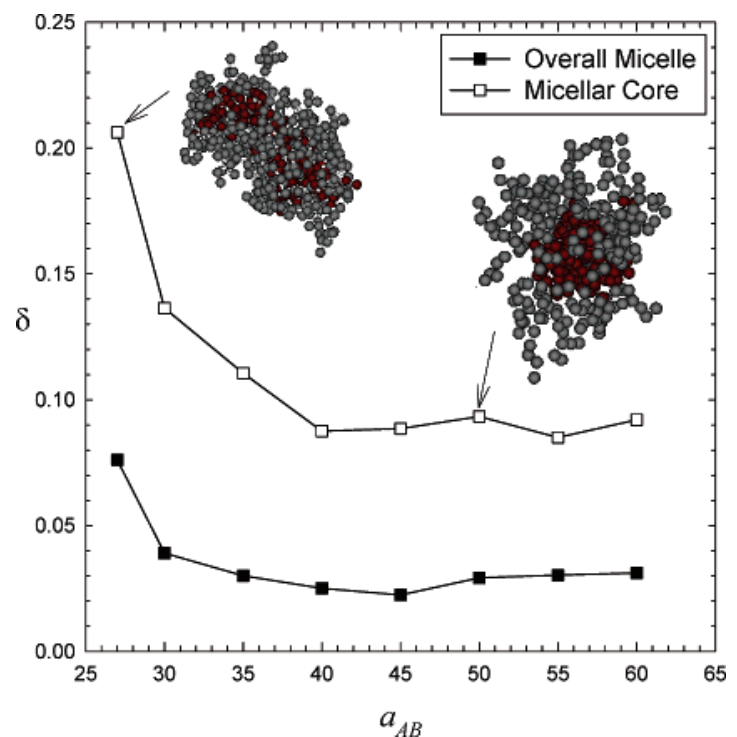

Figure 4. The asphericity is plotted against mutual compatibility $a_{\mathrm{AB}}$ for $A_{10} B_{5}$. (a) A snapshot of a typical core-corona micelle formed for incompatible $\mathrm{A}$ and $\mathrm{B}$ blocks $\left(a_{\mathrm{AB}}=50\right)$. (b) A snapshot of a micelle formed for compatible A and B blocks.

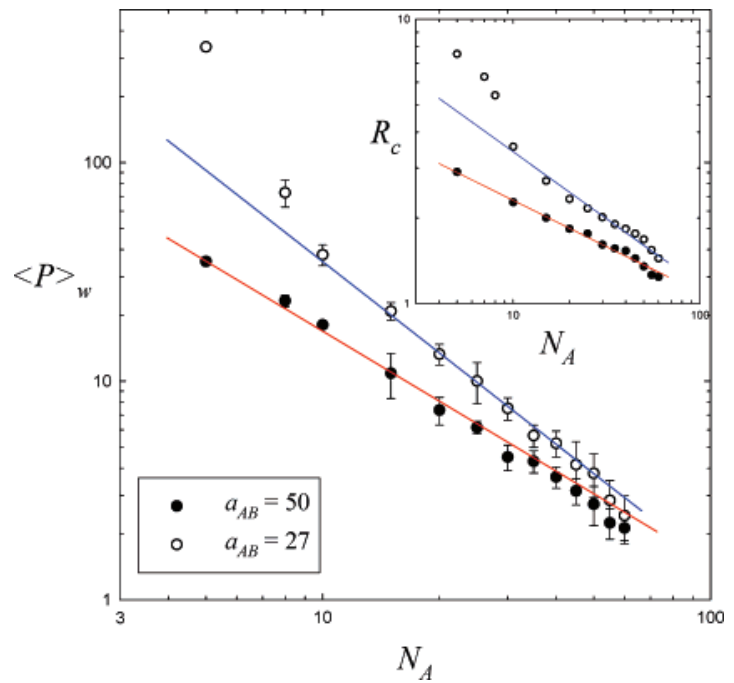

Figure 5. The variation of the weight-average aggregation number with the soluble-block length for mutually compatible $\left(a_{\mathrm{AB}}=27\right)$ and incompatible $\left(a_{\mathrm{AB}}=50\right)$ diblocks. In the inset, the micellar core radius is plotted against the soluble-block length.

surface free-energy is mainly caused by $a_{\mathrm{BS}}$ but not $a_{\mathrm{AB}}$. This statement is justified by realizing that micellization can be induced by large enough $a_{\mathrm{BS}}$ with small $a_{\mathrm{AB}}$ but not by large $a_{\mathrm{AB}}$ with small $a_{\mathrm{BS}}$. In fact, as $a_{\mathrm{AB}} \gtrsim a_{\mathrm{BS}}$, the micellar core prefers to contact the solvent rather than A blocks. In order to avoid core-corona contact, the elastic stretching of blocks A due to the repulsive interaction between like monomers may be further enhanced. As a result, the contribution of $a_{\mathrm{AB}}$ to the corona free-energy $\left(F_{\text {corona }}\right)$ is more than the surface energy $\left(F_{\text {interface}}\right)$. The corona free-energy can be lowered by reducing the aggregation number. On the other hand, as $a_{\mathrm{AB}} \lesssim a_{\mathrm{BS}}$, more core-corona contacts are favored at the cost of the deformation of the micellar shape from spherical shape.

C. Scaling Relations. In this work, the influence of A block size is examined by varying $N_{\mathrm{A}}$ from 5 to 60 for $N_{\mathrm{B}}=5$. Figure 5 shows the variation of $\langle P\rangle_{\mathrm{w}}$ with $N_{\mathrm{A}}$ for the conditions of mutual compatibility $\left(a_{\mathrm{AB}}=27\right)$ and incompatibility $\left(a_{\mathrm{AB}}=\right.$ 50) between $\mathrm{A}$ and $\mathrm{B}$ blocks. Our simulation results agree qualitatively with the model of Nagarajan and Ganesh. ${ }^{9}$ 


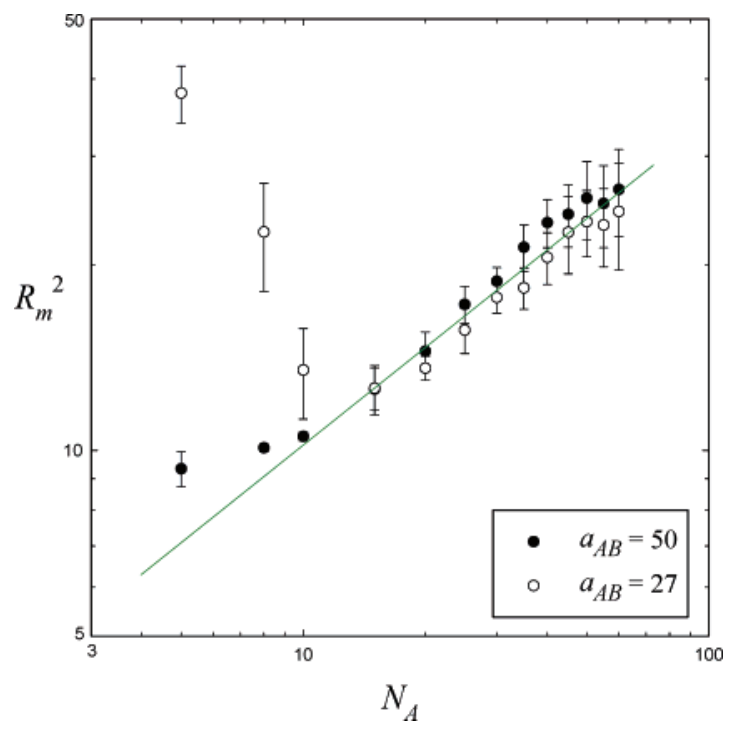

Figure 6. The variation of the micellar size $R_{\mathrm{m}}$ with the soluble-block length for mutually compatible $\left(a_{\mathrm{AB}}=27\right)$ and incompatible $\left(a_{\mathrm{AB}}=\right.$ 50) diblocks.

Evidently, the aggregation number decreases with the length of block A and it can be well represented by the following power law.

$$
\langle P\rangle_{\mathrm{w}} \sim N_{\mathrm{A}}^{-\alpha}
$$

Nevertheless, the exponent $\alpha$ is polymer specific and depends on the compatibility between $\mathrm{A}-\mathrm{B}$ blocks; for example, $\alpha=$ 1.05 for $a_{\mathrm{AB}}=50$ and $\alpha=1.41$ for $a_{\mathrm{AB}}=27$. This consequence can be attributed to the contribution of $\mathrm{A}-\mathrm{B}$ mutual compatibility to the free energy of the corona. Similarly, the micellar core radius declines weakly with A block size.

$$
R_{\mathrm{c}} \sim N_{\mathrm{A}}^{-\beta}
$$

If the solvophobic core is solvent-free and incompressible, it is anticipated $^{1}$

$$
P N_{\mathrm{B}} v_{\mathrm{B}} \approx \frac{4 \pi}{3} R_{\mathrm{c}}^{3}
$$

where $v_{\mathrm{B}}$ denotes the molecular volume of the B block. As a consequence, one has $R_{\mathrm{c}} \sim P^{1 / 3}$ and $\beta=\alpha / 3$. This statement is confirmed in the inset of Figure 5 for $R_{\mathrm{c}}$ versus $N_{\mathrm{A}}$. Our results show that $\beta=0.35$ for $a_{\mathrm{AB}}=50$ and $\beta=0.47$ for $a_{\mathrm{AB}}=27$.

Halperin obtained a scaling relationship for the overall radius in the starlike limit, $R_{\mathrm{m}} \sim N_{\mathrm{A}}^{3 / 5}$, due to the long chain behavior of A block. Nonetheless, our results of the $N_{\mathrm{A}}$ dependence of the aggregation number reveal that the micelle probably fails to form before the solvophilic block length reaches this limit. For example, as illustrated in Figure 5, $\langle P\rangle_{\mathrm{w}} \leq 2$ as $N_{\mathrm{A}} \geq 70$, indicating a solution with only dimers or single diblock copolymers. Figure 6 shows the variation of $R_{\mathrm{m}}$ with $N_{\mathrm{A}}$ and that the power law is followed for long A block, $R_{\mathrm{m}} \sim N_{\mathrm{A}}^{q}$, with $q \cong 0.29$. The fact that the exponent is less than $3 / 5$ in good solvents (and less than $1 / 3$ in poor solvents) indicates that the micelle does not possess the self-similar nature associated with three-dimension objects as $N_{\mathrm{A}}$ is increased. This consequence can be attributed to the fact that the extent of elastic stretching of blocks $\mathrm{A}$ in the corona rises with increasing the aggregation number. That is, for short soluble A blocks, the micelles are formed with large aggregation number, indicating the increment of the crowdedness in the corona. Thus, A blocks tend to stretch out resulting in a larger $R_{\mathrm{m}}$. For long soluble A blocks, the micelles are formed with small aggregation number and only a few A blocks in the corona. The A blocks can coil back freely. Therefore, the scaling relation for $R_{\mathrm{m}}$ and $N_{\mathrm{A}}$ is with an exponent a lot less than $3 / 5$. Our simulation results show that the expansion of the solvophilic blocks (corona thickness/ $N_{\mathrm{A}}$ ) increases as $N_{\mathrm{A}}$ declines.

The exponent $q$ associated with $R_{\mathrm{m}}$ scaling is expected to be different for different $a_{\mathrm{AB}}$. However, the leading behavior seems to be dominated by the A block length, and thus the effect of $a_{\mathrm{AB}}$ is not substantial when the scaling relation is followed. Figure 6 also shows that the deviation from the power law is obvious when the lengths of A and B block become comparable. For an incompatible diblock, $R_{\mathrm{m}}$ declines much slower than that given by the power law. This result reveals that the corona stretching is very significant for short A blocks in a crowded aggregate. For a compatible diblock, it is interesting to find that both micellar size and aggregation number actually grow rapidly with decreasing $N_{\mathrm{A}}$ (as $\left.N_{\mathrm{A}} \leq 10\right)$. It may be attributed to the substantial deformation from the spherical shape, which will be shown later.

Since the total micellar core-solvent interface is proportional to $R_{\mathrm{c}}{ }^{2} / P \sim N_{\mathrm{A}}^{\beta}$, the reduction of aggregation number actually leads to the increment of the free energy $F_{\text {interface. }}$ On the other hand, the repeated unit density of the solvophilic block, $N_{\mathrm{A}} P /$ $R_{\mathrm{m}}{ }^{3} \sim N_{\mathrm{A}}^{1-\alpha-3 q}$, declines with increasing $N_{\mathrm{A}}$, and this consequence indicates that reducing aggregation number yields translational entropy gain (dilution). Moreover, we have observed that the mean radius of gyration associated with each diblock copolymer in a micellar solution $\left\langle R_{\mathrm{g}}{ }^{2}\right\rangle_{\mathrm{m}}$ is always greater than that in an infinitely dilute solution $\left\langle R_{\mathrm{g}}{ }^{2}\right\rangle_{\mathrm{s}}$. For example, $\left\langle R_{\mathrm{g}}{ }^{2}\right\rangle_{\mathrm{m}} \simeq 1.46$ at $N_{\mathrm{A}}=5$ and $\left\langle R_{\mathrm{g}}{ }^{2}\right\rangle_{\mathrm{m}} \simeq 9.03$ at $N_{\mathrm{A}}=50$ for $a_{\mathrm{AB}}$ $=50$ while $\left\langle R_{\mathrm{g}}{ }^{2}\right\rangle_{\mathrm{s}} \simeq 1.09$ and 7.02 , respectively, for the corrsponding $N_{\mathrm{A}}$ values. This result points out that the formation of micelles leads to the stretch of the solvophilic block in the corona. Therefore, reducing the aggregation number gives conformation entropy gain (deformation). Since $\chi_{\mathrm{AS}} \simeq 0.3$ is small, the $N_{\mathrm{A}}$-dependence behavior reveals that the entropic contributions associated with A block dilution and deformation are responsible for the anticooperative process.

D. Micellar Morphology. A micelle is generally assumed to be spherical in shape. The insoluble blocks that form the spherical core are fully segregated from the soluble blocks that form the spherical corona. Such a micellar morphology can be examined in terms of the asphericity defined in eq 12. In general, this picture is expected to be true for incompatible $\mathrm{A}-\mathrm{B}$ diblocks. Figure 7 a shows the variation of the asphericity $\delta$ with the soluble-block length $N_{\mathrm{A}}$ for the incompatible diblock $a_{\mathrm{AB}}$ $=50$. For large $N_{\mathrm{A}}$ (e.g., $\left.\gtrsim 40\right)$, the core is quite loose and deviates significantly from the spherical shape because the aggregation number is small. Note that $\delta \approx 0.4$ for an isolated copolymer. As $N_{\mathrm{A}}$ decreases, the aggregation number rises and the core becomes more compact. The micellar shape approaches a sphere. When $N_{\mathrm{A}} \sim N_{\mathrm{B}}$, however, the asphericity is actually increased with decreasing $N_{\mathrm{A}}$. That is, the shape of the micellar core becomes like a spheroid even for the incompatible diblock. This consequence implies that it may be difficult to form a crewcut micelle with a spherical shape.

Figure $7 \mathrm{~b}$ illustrates the variation of $\delta$ with $N_{\mathrm{A}}$ for the compatible diblock $a_{\mathrm{AB}}=27$. The overall micelle (corona) is always more spherical than the core. Similar to the incompatible diblock, the asphericity generally declines as $N_{\mathrm{A}}$ decreases. When A block length is comparable to B-block length $\left(N_{\mathrm{A}} \leq\right.$ $10)$, however, $\delta$ grows rapidly but drops again at $N_{\mathrm{A}}=5$. The 

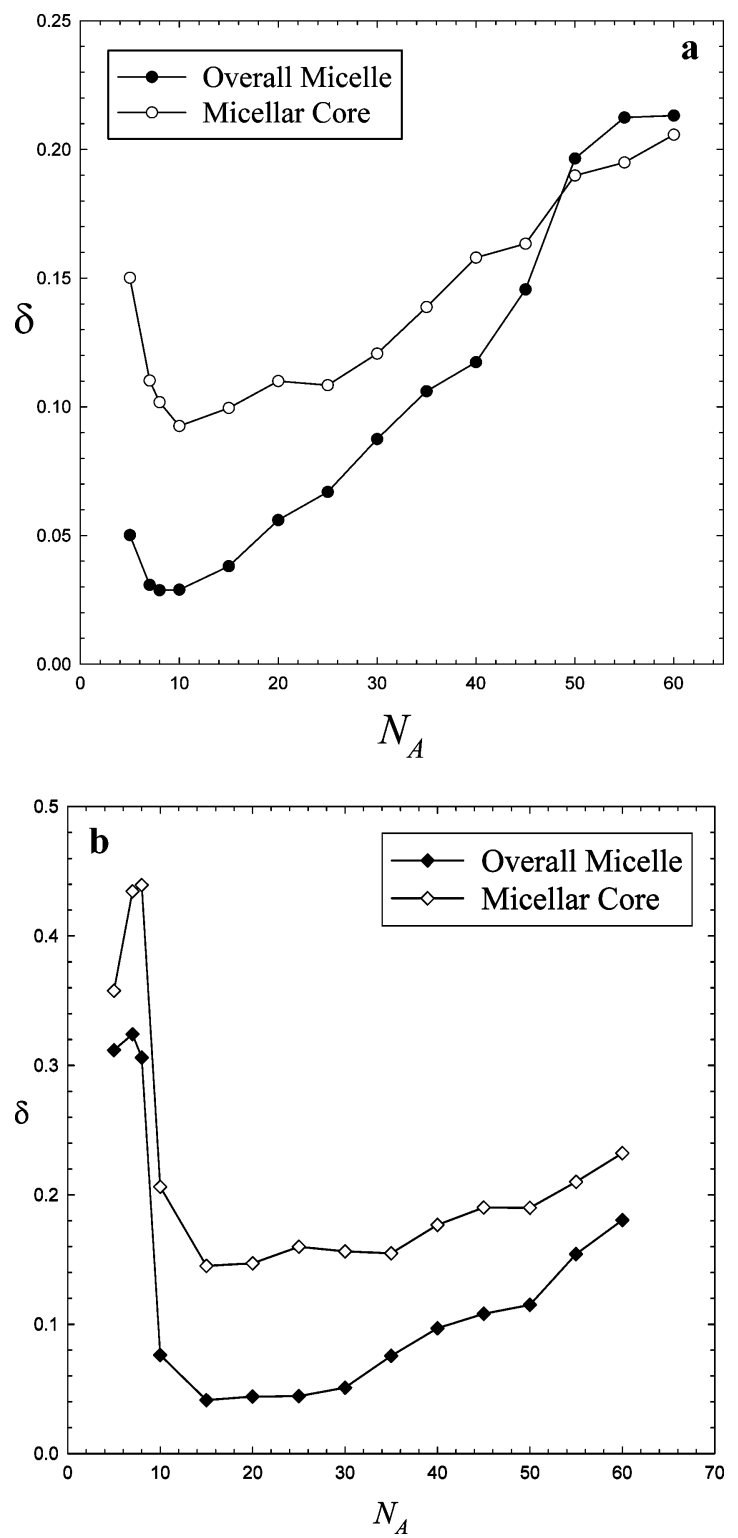

Figure 7. The micellar morphology is expressed in terms of the asphericity. The influence of soluble-block length on $\delta$ is given for (a) compatible diblock $\left(a_{\mathrm{AB}}=27\right)$ and $(\mathrm{b})$ incompatible diblock $\left(a_{\mathrm{AB}}=\right.$ $50)$.

$\delta$ value of the micellar core can be as large as 0.44 , indicating significant deviation from spherical shape. The typical morphologies of the overall micelle and micellar core are depicted in Figure $8 \mathrm{a}$ for $\mathrm{A}_{7} \mathrm{~B}_{5}$. One can clearly see that the micellar core displays a cylinder-like shape, which is able to accommodate a large amount of copolymers. It is quite surprising that the $\delta$ value falls back to 0.36 for the solvophobic core at even shorter soluble-block length $\left(\mathrm{A}_{5} \mathrm{~B}_{5}\right)$, which results in larger micelles. Figure $8 \mathrm{~b}$ illustrates the typical morphology associated with the insoluble B-block domain (the so-called core). The solvophilic blocks are observed to be entrapped in the solvophobic core. The holes within the core are actually occupied by the soluble A blocks. A reasonable explanation is given as follows: because soluble-block length is too short to protect the solvophobic core effectively, the mutual compatibility between A and B blocks allows the mixing of them to reduce the interfacial area between the solvophobic domain and solvent. A reduction in asphericity is therefore resulted. Our simulation results indicate that the

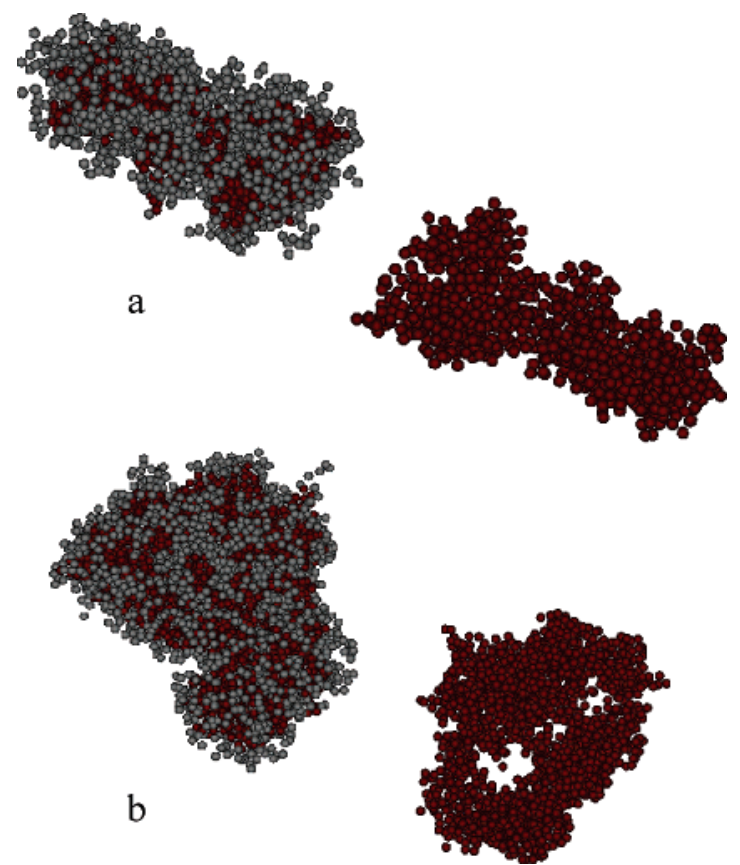

Figure 8. The typical snapshot of overall micelle and micellar core formed by (a) $\mathrm{A}_{7} \mathrm{~B}_{5}$ and (b) $\mathrm{A}_{5} \mathrm{~B}_{5}$ for incompatible diblock $\left(a_{\mathrm{AB}}=50\right.$ ). Note that the solvophilic A blocks are partially entrapped in the solvophobic $\mathrm{B}$ domains for $\mathrm{A}_{5} \mathrm{~B}_{5}$.

well-accepted core-corona scenario is not enough to depict the micellar morphology associated with $\mathrm{A}-\mathrm{B}$ compatible diblock copolymers.

In summary, on the basis of dissipative particle dynamics, the distribution of micellar size is obtained for diblock copolymers with different soluble-block lengths. We have shown that the weight-average aggregation number of micelles decreases with increasing the soluble-block length. The power law relation is followed but the exponent depends on the mutual compatibility between soluble and insoluble blocks. For the same composition, the incompatible diblocks form a smaller micelle and its aggregation number declines with a smaller exponent. Our results agree qualitatively with the theories of Nagarajan and Ganesh. ${ }^{9}$ The dependence of soluble-block length comes from the free energy of the corona, which is determined by the balance of the elastic stretching of blocks $\mathrm{A}$, the repulsive interaction between monomers, and the $\mathrm{A}-\mathrm{B}$ mutual compatibility. The analysis of micellar morphology in terms of asphericity points out the inadequacy of the spherical corecorona scenario, particularly for mutually compatible diblock copolymers.

Acknowledgment. This research is supported by the National Council of Science of Taiwan. Computing time, provided by the National Center for High-Performance Computing of Taiwan, is gratefully acknowledged.

\section{References and Notes}

(1) Hamley, I. W. The Physics of Block Copolymers; Oxford University Press: New York, 1998.

(2) de Gennes, P. G. In Solid State Physics; Liebert, L., Ed.; Academic Press: New York, 1978; Vol. 14.

(3) Daoud, M.; Cotton, J. P. J. Phys. 1982, 43, 531.

(4) Halperin, A. Macromolecules 1987, 20, 2943.

(5) Izzo, D.; Marques, C. M. Macromolecules 1993, 26, 7189

(6) Noolandi, J.; Hong, K. M. Macromolecules 1983, 16, 1443.

(7) Whitmore, M. D.; Noolandi, J. Macromolecules 1985, 18, 657

(8) Leiblert, L.; Orland, H.; Wheeler, J. C. J. Chem. Phys. 1983, 79, 3550 .

(9) Nagarajan, R.; Ganesh, K. J. Chem. Phys. 1989, 90, 5843. 
(10) Xu, R.; Winnik, M.; Riess, G.; Chu, B.; Croucher, M. D. Macromolecules 1992, 25, 644 .

(11) Lund, R.; Willner, L.; Stellbrink, J.; Radulescu, A.; Richter, D. Macromolecules 2004, 37, 9984.

(12) Tao, J.; Stewart, S.; Liu, G.; Yang, M. Macromolecules 1997, 30, 2738.

(13) Förster, S.; Zisenis, M.; Wenz, E.; Antonietti, M. J. Chem. Phys. 1996, 104, 9956.

(14) Willner, L.; Poppe, A.; Allgaier, J.; Monkenbusch, M.; Lindner, P.; Richter, D. Europhys. Lett. 2000, 51, 628.

(15) von Gottberg, F. K.; Smith, K. A.; Hatton, T. A. J. Chem. Phys. 1997, 106, 9850.

(16) Milchev, A.; Bhattacharya, A.; Binder, K. Macromolecules 2001, 34,1881 .

(17) Sheng, Y.-J.; Nung, C.-H.; Tsao, H.-K. J. Phys. Chem. B 2006, 110, 21643.
(18) Chou, S.-H.; Tsao, H.-K.; Sheng, Y.-J. J. Chem. Phys. 2006, 125 194903.

(19) Hoogerbrugge, P. J.; Koelman, J. M. V. A. Europhys. Lett. 1992, 19,155

(20) Groot R. D.; Warren, P. B. J. Chem. Phys. 1997, 107, 4423.

(21) Español, P.; Warren, P. B. Europhys. Lett. 1995, 30, 191.

(22) Rudnick, J.; Gaspari, G. J. Phys. A: Math Gen. 1986, 19, L191193.

(23) Zhou, T.; Chen, S. B. Macromolecules 2005, 38, 8554.

(24) Noguchi, H.; Yoshikawa, K. J. Chem. Phys. 1998, 109, 5070.

(25) Won, Y. Y.; Davis, H. T.; Bates, F. S. Macromolecules 2003, 36 , 953.

(26) Kumar, S. K.; Floriano, M. A.; Panagiotopoulos, A. Z. Adv. Chem. Eng. 2001, 28, 260

(27) Panagiotopoulos, A. Z.; Floriano, M. A.; Kumar, S. K. Langmuir 2002, 18, 2940 . 